\title{
O ALIVIO DA DOR DE PACIENTES NO PÓS-OPERATÓRIO NA PERSPECTIVA DE ENFERMEIROS DE UM CENTRO DE TERAPIA INTENSIVA*
}

\author{
POSTOPERATIVE PATIENT'S PAIN RELIEF UNDER PERSPECTIVE OF \\ NURSES OF THE INTENSIVE CARE UNIT
}

\author{
EL ALIVIO DEL DOLOR EN PACIENTES DURANTE EL POST OPERATORIO \\ EN LA PERSPECTIVA DE ENFERMEROS DE UN \\ CENTRO DE TRATAMIENTO INTENSIVO
}

Vanessa da Silva Carvalho Vila** Fernanda Carneiro Mussi***

\begin{abstract}
VILA VSC, MUSSI FC. O alívio da dor de pacientes no pós-operatório na perspectiva de enfermeiros de um centro de terapia intensiva. Rev Esc Enferm USP 2001; 35(3):300-7.

RESUMO

O presente estudo teve como objetivo identificar como os enfermeiros aliviam a dor de pacientes durante o pós-operatório, no Centro de Terapia Intensiva de um hospital geral, de grande porte, localizado no municipio de São Paulo-SP Participaram 14 enfermeiros, com idade entre 20 e 40 anos, em que 78,6\% eram do sexo feminino e 42,9\% tinham de 1 a 5 anos de atuação profissional. Os dados foram coletados através de entrevista semi-estruturada, gravada e analisados qualitativamente, utilizando-se as etapas da análise de conteúdo, propostas por Bardin(1). Duas categorias principais foram evidenciadas: avaliação da dor e condutas tomadas para alivio da dor. Os resultados mostraram que os enfermeiros utilizam tanto a terapêutica medicamentosa como a complementar para o alivio da dor e consideram a sua avaliação como conduta inicial para estabelecer ações de prevenção e alivio deste desconforto.
\end{abstract}

PALAVRAS-CHAVE: Dor. Enfermagem. Unidades de Terapia Intensiva. Cuidados de conforto.

\begin{abstract}
The objective of the present study was to identify how nurses releave the post-operative patients pain in the Intensive Care Unit of a general hospital, situated in São Paulo city concil. Participated of this study 14 nurses between 20 and 40 years, and 78,6\% were females and 42,9\% had 1 to 5 years of professional performance. The data were collected through halfestructured recordered interview and qualitativelly analysed, applying the stages proposed by Bardin(1) . Two categories were found: appraisement of pain and conducts to releave pain. The results displayed that the nurses used as the medicinal therapeutics as the complementaries to pain relief an considered the appraisement of this pain as initial conduct to set up a way to comfort, prevention and relief.
\end{abstract}

KEYWORDS: Pain. Nursing. Intensive Care Units. Comfort care.

\section{RESUMEN}

Este estudio tuvo como objetivo identificar cómo los enfermeros alivian el dolor de pacientes durante el post-operatorio, en el Centro de Tratamiento Intensivo de un hospital general de gran porte, ubicado en el municipio de São Paulo-SR Participaron 14 enfermeros, con edad entre 20 y 40 años, de los que un 78,6\% eran del sexo femenino y un $42,9 \%$ tenian de 1 a 5 años de experiencia profesional. Los datos fueron colectados a través de entrevista semistructurada, grabada y analizada cualitativamente, utilizando las etapas del análisis de contenido, propuestas por Bardin (1) . Das categorias principales fueron evidenciadas: evaluación del dolor y conductas tomadas para aliviar el dolor. Los resultados muestran que los enfermeros utilizan tanto el tratamiento medicamentoso como el complementar para el alivio del dolor y consideran su evaluación como conducta para establecer acciones de preventión y alivio de este desconforto.

PALABRAS-CLAVE: Dolor. Enfermerfa. Unidade de Terapia Intensiva. Conforto.

Trabalho para obtenção do titulo de especialista em Enfermagem em Terapia Intensiva na Faculdade de Enfermagem do Hospital Israelita Albert Einstein, São Paulo-SP, 1998.

** Enfermeira. Mestre em Enfermagem. Profa. Assistente do Departamento de Enfermagem da Universidade Católica de Goiás.

*** Enfermeira. Doutora em Enfermagem. Assistente de Coordenação do Uni-FMU. Professora da UNICSUL. vscvila@uol.com.br 


\section{INTRODUÇÃO}

Uma pessoa em estado crítico está sujeita a vivência de inúmeros desconfortos que vão desde a condição de adoecer até os processos de diagnóstico e intervenção médico-hospitalar.

Em nossa experiência profissional, prestando assistência de enfermagem a pacientes internados em Centros de Terapia Intensiva (CTI), durante o pósoperatório de cirurgias de médio e grande porte, entre elas, cardiacas, abdominais e vasculares, observamos a freqüente queixa de desconforto ocasionado pela dor, de natureza aguda. Além disso, a análise da literatura mostra que o ambiente do CTI caracteriza-se como estressante e o tratamento como invasivo para o paciente, constituindo-se num dos principais agravantes para a ocorrência de dor e desconforto(2-3)

A presença de dor acarreta aumento substancial do desconforto, além de freqüentemente o paciente em estado critico estar impossibilitado ou com dificuldade de comunicar seu sofrimento pelo uso freqüente e imperativo de artefatos e restrições impostas pelos métodos terapêuticos ${ }^{(4)}$ Desse modo, o alivio da dor e a promoção de conforto para os pacientes no Centro de Terapia Intensiva (CTI) deve ser prioritário, não apenas por razões humanas e éticas, mas também pela melhora que produz no estado fisico, mental e social do paciente.

Dentre os inúmeros conceitos referentes à dor encontrados na literatura, temos a definição proposta por Eisenach(5) de que, dor é uma sensação fisica, dificil de ser suportada, sofrimento, angústia do corpo ou do espírito, uma situação traumática geradora de angústia. A Associação Internacional para o Estudo da Dor (IASP) a define como "uma sensação desagradável e uma experiência emocional em resposta a uma lesão tecidual real ou potencial, ou descrita em tais termos".

Pimenta(4) relaciona a dor a uma "experiência complexa e subjetiva, cuja percepção não é, meramente, o resultado da quantidade de tecido lesado, mas envolve um mecanismo complexo, determinado por muitos fatores, incluindo idade, sexo, cultura, influências ambientais e múltiplas variáveis psicológicas e sociais". Para a autora todos esses fatores devem ser explorados para que a avaliação da dor seja completa. "Acreditar na queixa do paciente e reconhecer o efeito devastador que a dor pode ter sobre o mesmo são pontos fundamentais, não somente para avaliar o quadro álgico, mas também para o seu manejo adequado".

A dor é classificada como aguda e crônica. Dor aguda é definida como a dor de início recente e provavelmente de duração limitada (6) Geralmente é possivel ser identificada uma causa relacionada a uma injúria ou doença. A dor pós-operatória é classificada como dor aguda, sendo uma combinação de lesão tecidual, dor $t$ ansiedades ${ }^{(7)}$.
Entre as dores agudas de maior incidência no âmbito hospitalar, encontramos a manifestada por pessoas submetidas às mais diversificadas intervenções cirúrgicas ${ }^{(8)}$, o que vem de encontro com a observação feita em nossa prática de enfermagem. A ocorrência de dor pós-operatória depende da influência de fatores culturais, psicológicos como ansiedade, depressão, medo, entre outros, da extensão do trauma durante a intervenção cirúrgica, da habilidade técnica do cirurgião, das doenças prévias, do local e do tipo da incisão. Atualmente, sabe-se que as diferenças individuais estão relacionadas aos processos de modulação da dor ao nivel do sistema nervoso central, as quais podem explicar porque sua intensidade varia, de individuo para individuo, quando intervenções semelhantes são realizadas $(5,6,9)$

Embora seja destacada a necessária competência do enfermeiro e de outros profissionais no manejo da dor, a Sociedade Americana de Dor (1992) relata a deficiência da equipe multiprofissional para avaliar e aliviar a dor, como razão mais comum para que pacientes hospitalizados não tenham alivio da dor nos Estados Unidos da América. Assim, o pouco conhecimento por parte dos enfermeiros sobre a dor e seu tratamento pode constituir-se numa das principais barreiras para promoção de conforto nessa situação (10)

O enfermeiro assume papel preponderante na avaliação precisa da experiência dolorosa e na promoção, manutenção e restauração do conforto frente a vivência da dor e suas conseqüências. Kubecka (10) e Bernardo (11) enfatizam que os enfermeiros têm a responsabilidade profissional e ética de manejar a dor e aliviar o sofrimento. Murray (2) corrobora com a importância do alivio da dor, ao afirmar que o tratamento da dor no CTI requer conhecimento dos mecanismos nociceptivos e do impacto da dor sobre o bem-estar do paciente, bem como, o comprometimento profissional com o tratamento adequado da dor.

O processo de avaliação da dor é amplo e envolve a obtenção de informações relacionadas à data de início, localização, intensidade, duração e periodicidade dos episódios dolorosos; qualidades sensoriais e afetivas do paciente; fatores que iniciam, aumentam ou diminuem a sua intensidade; significado para o paciente e sua família; interferência nas atividades de vida diária, nos relacionamentos afetivos e no trabalho; expectativas em relação à doença e ao tratamento; comportamento habitual em situações de estresse; tipos e resultados de tratamentos anteriormente realizados (4)

Segundo Kubecka (10), o alivio da dor é um prérequisito para que o paciente obtenha uma ótima recuperação e qualidade de vida. Algumas das conseqüências para o paciente, sem alivio da dor, 
incluem sofrimento, ansiedade, medo, depressão, raiva, imobilidade e isolamento e, tais conseqüências, podem contribuir para um decréscimo da qualidade de vida.

Considerando a importância do manejo adequado da dor como atividade do enfermeiro, a natureza estressante e invasiva do tratamento do paciente crítico constituindo-se num dos agravantes principais para a ocorrência de dor e desconforto e, nossa vivência em CTI, observando a queixa de dor aguda por parte de pacientes em pós-operatório de cirurgias de médio e grande porte, o presente estudo teve como preocupação e interesse identificar como os enfermeiros que atuam no CTI aliviam a dor dos pacientes durante o pós-operatório.

\section{OBJETIVO}

Identificar como os enfermeiros que atuam em um Centro de Terapia Intensiva, aliviam a dor de pacientes durante o pós-operatório.

\section{MATERIAL E MÉTODO}

O trabalho foi realizado no CTI de um hospital particular, geral, de grande porte, localizado no município de São Paulo - SP. O CTI é um complexo de internação, composto por 75 leitos, 30 destinados à terapia intensiva e 35 à semi-intensiva. A escolha da referida unidade relacionou-se ao fato de que a maioria dos pacientes submetidos a cirurgias de médio e grande porte são ali internados no período pós-operatório imediato, o qual se relaciona com alta incidência de dor aguda.

Do total de enfermeiros que atuam neste CTI, $35 \%$ foram sorteados dos plantões diurno, vespertino e noturno. Assim, fizeram parte da amostra 14 enfermeiros, com idade entre 20 a 40 anos, 78,6\% eram do sexo feminino e $42,9 \%$ tinham entre 1 a 5 anos de atuação profissional.

Para coleta de dados foi elaborado um instrumento composto por duas partes. A primeira constituída por questões que levantaram dados de identificação dos enfermeiros quanto ao sexo, idade e tempo de atuação profissional e, a segunda, por um questão norteadora que levantou dados sobre como os enfermeiros aliviam a dor dos pacientes no periodo pós-operatório.

A coleta de dados foi realizada no período de dezembro de 1997 à março de 1998 após autorização da Chefia de Enfermagem do CTI. A técnica para coleta de dadas foi a entrevista, a qual foi gravada, realizada individualmente, em ambiente privativo, por uma das autoras, com prévio consentimento dos enfermeiros. Os enfermeiros foram orientados quanto aos objetivos da pesquisa e assegurados do seu anonimato e direitos enquanto sujeitos de pesquisa. A questão norteadora do estudo foi: Como você alivia a dor do paciente no periodo pós-operatório?

Para análise dos dados foram utilizadas as etapas para análise de conteúdo propostas por Bardin (1) Inicialmente, as entrevistas gravadas foram transcritas na integra. Procedeu-se, posteriormente, a leitura flutuante e o recorte do texto em núcleos de sentido, isto é, o recorte de ordem semântica. Uma vez identificados os núcleos de sentido fez-se o agrupamento daqueles que indicavam um mesmo fenômeno emergindo. O processo de agrupar núcleos de sentido que pertencem aos mesmos fenômenos é chamado categorização. Desse modo emergiram categorias e sub-categorias que expressaram as ações dos enfermeiros para aliviar a dor do paciente no pósoperatório.

\section{RESULTADOS}

Duas categorias principais foram evidenciadas a partir da análise dos dados, as quais foram denominadas: avaliação da dor e condutas tomadas para alivio da dor.

A categoria avaliação da dor abrange subcategorias denominadas: avaliação das características da dor, consideração de fatores que influenciam a avaliação da dor, avaliação de sinais indiretos de dor, avaliação das conseqüências da dor, avaliação de fatores que geram dor" e o papel da enfermagem na avaliação da dor.

A avaliação das características da dor retratou que os enfermeiros têm como ação inicial para alivio da dor e promoção de conforto a identificação da presença, do local, da intensidade e do tipo de dor.

Os enfermeiros julgaram importante para o alivio da dor a consideração de fatores que influenciam a avaliação da dor os quais foram relacionados à capacidade do paciente em verbalizar a dor, à subjetividade característica da dor e às características pessoais na manifestação da dor.

Outro aspecto apontado pelos enfermeiros em relação a avaliação da dor, foi associado a importância da avaliação de sinais indiretos de dor, pelo fato de que, muitas vezes, o paciente no CTI está impossibilitado de se comunicar verbalmente, principalmente, devido a entubação oro-traqueal e traqueostomia, entre outras razões.

Os enfermeiros focaram também sua ação na avaliação das conseqüências da dor e as relacionaram 
a ansiedade, irritação, alterações fisiológicas como taquicardia e hipertensão, alterações comportamentais e a dificuldade do paciente encontrar uma posição confortável.

Ao se referirem a avaliação dos fatores que geram dor, consideraram em seu julgamento a presença de procedimentos e equipamentos, a incisão cirúrgica, o posicionamento, o barulho e o estado emocional do paciente.

Ainda no que se refere às percepções dos enfermeiros sobre a avaliação da dor, salientaram $\mathrm{O}$ papel da enfermagem na avaliação da dor, por serem profissionais que estão mais próximos do paciente e os principais responsáveis pela avaliação da dor, atuando como intermediários entre o médico e o paciente.

$\mathrm{Na}$ categoria condutas para alivio da dor os enfermeiros relataram que procuravam aliviar a dor por meio de diferentes ações e estratégias de interação com o paciente e intervenções sobre o ambiente hospitalar. Várias subcategorias expressaram estas condutas: promover o conforto, tocar e conversar com o paciente, minimizar o barulho, evitar negar a dor, focar atenção no paciente, valorizar e partilhar a dor, evitar a manipulação do paciente, ouvir o paciente, explicar o motivo da dor, medicar, desviar a atenção do paciente da dor, mudar a posição do paciente, oferecer apoio psicológico e orientar quanto as medidas tomadas para alivio da dor. Verbalizaram, também, a importância da prescrição eficaz de analgésicos pelo médico para o adequado alivio da dor.

No decorrer da análise emergiram subcategorias relacionadas a condutas para alivio da dor que refletiram a percepção dos enfermeiros sobre como a dor vem sendo considerada nos seus contextos de atuação profissional e sobre possiveis medidas terapêuticas que poderiam ser adotadas para seu alivio. Embora tais dados não se relacionem diretamente aos objetivos deste estudo, são aqui apresentados, pois acreditamos que têm implicações para a assistência, ensino e pesquisa de enfermagem. Os enfermeiros colocaram a importância da analgesia controlada pelo paciente como um tratamento recente no manejo da dor e consideraram este método de imprescindivel ajuda para a promoção de um pósoperatório mais confortável. Outra questão apontada por eles foi a subestimação da dor, principalmente por médicos, enfermeiros e auxiliares de enfermagem. Os enfermeiros relataram que muitas vezes a avaliação da dor e seu alivio são objetivos secundários da assistência. Destacaram, também, a falta de ações para a prevenção de dor, esperando-se o paciente sentir dor para depois tratá-la com medicamentos.

\section{DISCUSSÃO}

Segundo Pimenta (4) a dor é uma manifestação clínica que surge com freqüência, sendo a sua avaliação uma tarefa complexa para o enfermeiro que presta cuidados ao paciente. Tal como descrito na literatura, observou-se neste estudo a preocupação dos enfermeiros no processo de avaliação da dor, a qual é expressa, entre outras, na subcategoria avaliação das caracteristicas $d a$ dor e seus respectivos núcleos de sentido: "perguntar se o paciente tem dor", descobrir o local da dor", "avaliar a intensidade da dor" e "analisar o tipo de dor do paciente". Piment(12), retratam que a identificação e avaliação da dor são os passos mais importantes no direcionamento da intervenção de enfermagem com o objetivo de minimizar o sofrimento de pacientes com dor. Leisfer (3) salienta que a avaliação da dor consiste de descrições objetivas, subjetivas e verbais do paciente, sendo necessário anotar o aparecimento, a duração, a qualidade e as verdadeiras palavras do paciente descrevendo a dor.

Referente ao processo de avaliação da dor, os enfermeiros expressaram fatores que o influenciam, exemplificados por núcleos de sentido como: "achando que o paciente precisa estar orientado para avaliar a dor", "achando a avaliação da dor muito subjetiva", observando que o paciente oriental fica mais na dele". Estes fatores se relacionaram, respectivamente, à capacidade do paciente em verbalizar a dor, à subjetividade da dor e às características pessoais na manifestação da dor.

Em relação a capacidade do paciente verbalizar a dor, Pimenta (4) afirma que "muitas vezes a possibilidade do paciente comunicar a dor está diminuída; a comunicação verbal fica prejudicada ou completamente abolida pelo uso de artefatos como cânulas de entubação, de traqueostomia, de guedel, entre outras. As restrições fisicas, ocasionadas pelos eletrodos, catéteres e sondas, ou mesmo pela contenção de mãos, acabam interferindo na comunicação nãoverbal". Neste sentido, os participantes deste estudo colocaram que "acham mais fácil avaliar a dor do paciente que verbaliza" demonstrando que a impossibilidade de verbalizá-la dificulta a sua avaliação.

No que se refere à subjetividade característica da dor como fator que influencia a sua avaliação, Bonnet (13) refere que não há relação previsivel entre lesão e dor, e o caráter subjetivo desta torna difícil sua avaliação. Cada experiência dolorosa do indivíduo é influenciada por sua própria história pessoal, pela compreensão que ele tem da dor e por seu estado mental. Pessoas em condições idênticas ou similares podem se comportar de modo diferente por causa da variação de personalidade e da experiência. 
Melzack, Wall (7) mostraram que a dor é muito mais variável e modificável do que muitos acreditavam no passado diferindo de pessoa para pessoa, de cultura para cultura. O caráter subjetivo da dor e sua avaliação é evidenciado, também, no conceito proposto por Sofaer (14): "dor é o que o paciente diz ser e existe quando ele diz existir". Alguns enfermeiros podem sentir dificuldade em acreditar nas afirmações de um paciente a respeito da dor, mas, apesar de não termos evidências objetivas para o seu diagnóstico, devemos confiar no que o paciente nos diz sentir.

Quanto as características pessoais influenciando a avaliação da dor os enfermeiros expressaram o aspecto cultural: "achando que é cultural o paciente não se queixar de dor". Segundo Jimenez ${ }^{(15)}$ atualmente há certos grupos étnicos que ainda ligam a dor a punição divina e alguns religiosos consideram aceitáveis a sua própria dor e sofrimento como significado de uma purificação e crescimento espiritual. Em algumas culturas, os rituais, que para alguns podem ser associados a desconforto extremo, não parecem causar problemas para as pessoas envolvidas, enquanto outros estímulos produzem uma resposta dolorosa acentuada (16) demonstraram que os niveis de tolerância à dor realmente variam de uma cultura para a outra.

$\mathrm{Na}$ subcategoria avaliação de sinais indiretos de dor, os enfermeiros ressaltaram a taquicardia e a hipertensão como possiveis sinais de dor em pacientes entubados e que não conseguem falar. Gozzani (17) afirma que a dor tem repercussões fisiológicas genericamente referidas como "respostas ao estresse" que atualmente são vistas como respostas de não adaptação e que podem gerar sérias complicações ao paciente crítico. Segundo esse autor, a dor pode desencadear reações reflexas segmentares e suprasegmentares, que incluem distúrbios na função pulmonar, cardiovascular, gastrointestinal e urinária, prejuízo da função e metabolismo muscular, bem como neuroendócrino, alterações metabólicas e imunológicas. Os enfermeiros precisam estar atentos e tornarem-se capacitados para a avaliação de indicadores não verbais de dor que incluem, alteração dos sinais vitais, modificação das características da pele, náuseas, vômitos, comportamentos como choro e lamentação, palidez, agitação, entre outros. Os dados objetivos indicadores de dor podem incluir diaforese, taquicardia, alteração na pressão arterial e dilatação da pupila. No CTI precisa-se saber diferenciar esses sintomas associados à dor daqueles decorrentes de outras patologias (31)

Observou-se nesta investigação, que os enfermeiros valorizam a avaliação de fatores que geram dor, considerando os procedimentos a que o paciente é submetido, os equipamentos que faz uso, a incisão cirúrgica, a posição, o barulho e o estado emocional. Com relação a procedimentos e equipamentos como causa de dor, observou-se núcleos de sentido como: "sabendo que procedimentos muito invasivos geram dor", verificando que às vezes o que dói é um tubo, um catéter". Segundo Murray(2), muito daquilo que realizamos na UTI está associado à dor, seja a cateterização venosa central, a entubação, a sondagem nasogástrica, com a conseqüente presença de uma estrutura estranha irritante, às vezes durante dias Mesmo um procedimento de rotina como a percussão torácica pode ser bastante doloroso para muitos pacientes. Assim, aquilo que foi feito para promover conforto acaba sendo a própria fonte de desconforto, mesmo que seja temporário.

Com relação a incisão cirúrgica, foram extraídos núcleos de sentido como: "descobrindo se é realmente a incisão que dói", " procurando saber se a dor é da incisão cirúrgica". Pimenta (12) afirmam que "toda intervenção cirúrgica traumatiza tecidos, a dor resultante depende do local e do tipo de cirurgia. A resposta inflamatória à cirurgia libera histaminas, serotoninas, cininas e prostaglandinas, todas estimulantes dos receptores da dor. Além disso, há secção de nervos e espasmos musculares, levando a isquemia, acidose e hipóxia local, e conseqüentemente, a estimulação dos receptores de dor."

Quanto ao estado emocional dos pacientes os enfermeiros referiram a "importância de estar atento para o lado psicológico e de se verificar até que ponto a dor pode ser ansiedade". Segundo Murraiy(2), a admissão em uma UTI é por si só provocadora de ansiedade, e o grau de ansiedade do indivíduo pode determinar aumento na percepção da dor. Bond (18) retrata que os pacientes com dor, demonstram alterações emocionais como apreensão, pânico ou mesmo terror e podem tornar-se solitários e deprimidos ou apresentar niveis aumentados de tensão emocional, acompanhados por crises de irritabilidade e intolerância aos que o cercam. Meinhart (19), McCaffery (20) também ressaltam que a ansiedade é a sensação mais freqüentemente associada à dor aguda, sendo reconhecida como responsável pelo aumento da percepção dolorosa. A ansiedade está relacionada à intensidade da dor e aos possiveis significados que a dor tem para o paciente.

\section{$\mathrm{Na}$ categoria condutas tomadas para alivio}

da dor, os enfermeiros relataram medidas medicamentosas, mas também enfatizaram condutas independentes da intervenção médica. Observou-se que utilizam medidas alternativas descritas na literatura que visam promover relaxamento e distração e, consequentemente, podem permitir ao paciente sentir-se mais confortável. Algumas ações dos enfermeiros estão refletidas nos seguintes núcleos de sentido:" "perguntar se o paciente quer assistir televisão, "tentar deixar o paciente numa posição confortável", 
"deixar o paciente descansar bastante", "conversar com o paciente".

A farmacologia parece a melhor forma para controlar e combater a dor, porém existem outras medidas que podem ser usadas pela enfermagem, que independem da prescrição médica. Os enfermeiros podem lançar mão de técnicas de relaxamento e distração proporcionando ao paciente uma maior sensação de controle da dor. Sofaer (14) menciona que a distração consiste em focalizar atenção em outros estimulos entre eles, a televisão, música, figuras, livros e artes manuais. Essa técnica é especialmente útil, quando se deseja executar um procedimento doloroso, como a troca de um curativo, ou durante o período em que se espera pelo efeito analgésico de medicamentos administrados. O enfermeiro pode, também, transmitir ao paciente que está ciente de sua dor e que deseja efetuar mudanças na assistência para minimizá-la.

Os enfermeiros também identificaram o toque como medida para aliviar a dor e promover o conforto: "achando que o toque alivia a dor, "fazendo massagem para ver se melhora a dor e conforta". Harrison (21) mencionam que a terapia do toque tem sido utilizada com sucesso para aliviar a dor em pacientes críticos. Os enfermeiros usam alguns princípios e técnicas de terapia do toque quando realizam massagem nas costas, seguram a mão ou massageiam a área dolorosa. Esta intervenção é eficaz na redução da ansiedade e controle da dor.

Outro importante aspecto observado quanto a condutas para alivio da dor foi relacionado a explicação do motivo da dor , a orientação quanto as medidas tomadas para alivio da dor e a valorização da dor referida pelo paciente como ilustram os núcleos de sentido: "informar ao paciente que medicamentos ele está recebendo", "conforto é o paciente saber que você está procurando melhorar a sua dor, para que ele não tenha dor de forma nenhuma", "considerar que o paciente não tem que sentir dor". Com isto notouse que os enfermeiros estão atentos a necessidade de informar ao paciente seus esforços para prevenir e sanar a dor e que preocupam-se com o seu conforto. Leisfer (3) defende que oferecer informações pode ser uma estratégia para reduzir a dor. Os pacientes precisam ser informados acerca dos procedimentos, de seu impacto sobre a assistência, dos efeitos das medicações, etc. Isso poderá ajudar a reduzir a dor e aliviar a ansiedade. Os enfermeiros relataram que é possivel ajudar o paciente dando informações e ensinando técnicas de relaxamento. Além disto, em termos de redução da ansiedade e conseqüentemente diminuição da dor pós-operatória, os pacientes podem beneficiar-se da aquisição de informações préoperatórias.
Os enfermeiros destacaram, também, a importância da adequada intervenção médica para alivio da dor, principalmente no que diz respeito a prescrição de analgésicos. Donavan (22) observaram que quando as enfermeiras sugeriam uma intervenção para controle da dor, um analgésico era o tratamento mais freqüentemente proposto. Embora, neste estudo, tenha sido constatado que os enfermeiros lançam mão de diferentes medidas para alivio da dor e não exclusivamente da terapêutica medicamentosa, vários núcleos de sentido apontaram, também, para a valorização do uso e avaliação da eficácia dos analgésicos: " reforçar para o médico quando a dor não passou para ele dar algo mais forte", "procurar o médico para prescrever analgésico", pedir ao plantonista reavaliar o analgésico quando não se considera eficaz". Pereira (8) coloca que o uso de analgésicos é uma das formas mais utilizadas para analgesia da dor no meio clínico e deve ser realizada avaliando-se inicialmente a intensidade, as qualidades e o tipo de dor sentida. $O$ analgésico de escolha deve ser o mais eficaz e o mais bem tolerado pelo paciente.

Segundo Eisenach(5), Ready (6), o insucesso em proporcionar alivio adequado da dor no pós-operatório é devido, sobretudo, à dificuldade em se modificar intervenções inadequadas decorrentes da falta de conhecimento dos profissionais da área em relação à avaliação da dor, às doses efetivas de analgésicos, ao tempo de ação dessas drogas e aos seus efeitos colaterais. No caso dos narcóticos, por exemplo, medos infundados em relação ao desenvolvimento de vício e depressão respiratória levam à subprescrição e subministração de tais drogas.

A análise dos depoimentos evidenciou subcategorias referentes as concepções dos enfermeiros relacionadas a dor e às medidas para aliviá-la e sua visão sobre como a dor é considerada pelos profissionais de saúde, no dia-a-dia, no CTI. Um dos aspectos recentes no tratamento da dor é a utilização da analgesia controlada pelo paciente (PCA), e nas falas dos participantes haviam núcleos de sentido salientando o PCA como : " uma Ajuda imprescindivel para alivio da dor e para que o paciente tenha um pós-operatório mais tranqüilo e confortável". Pimenta (12) relatam que a PCA consiste no processo de utilização de bombas de infusão especiais programáveis e acionadas pelo próprio paciente, no momento da dor. Harrison (21) enfocam que a PCA permite a infusão contínua de pequenas quantidades de narcóticos promovendo um nivel mais constante de medicação e, assim, de analgesia. Esse recurso terapêutico, também, permite que o paciente administre pequenas dosagens da medicação, de acordo com a sua própria avaliação da dor. Estudos evidenciam que os pacientes requerem menores quantidades de analgésicos quando podem controlar a sua administração. 
Os enfermeiros também levantaram que muitas vezes a dor é subestimada, principalmente, por médicos, enfermeiros e auxiliares de enfermagem: "muitas vezes a avaliação da dor e seu alívio são vistos como um aspecto secundário", "outras coisas são priorizadas em relação à dor". Salientaram que pouco se preveni a dor: "espera-se o paciente ter dor para depois tratá-la de maneira medicamentosa". Pimenta (12) reforçam a expressão desses enfermeiros relatando que "muitas vezes o alivio da dor não se constitui prioridade de cuidado na concepção de muitos profissionais de terapia intensiva. Aceita-se a existência de dor como um fato consumado". McCaffery (20) também corrobora com esta percepção ressaltando que muitos enfermeiros optam pela administração do analgésico prescrito se necessário, isto é, esperam que o paciente apresente dor intensa para então medicar.

No entanto, a literatura atual defende que o controle da dor deve ser preventivo, que a dor não deve retornar com medidas eficazes ou pelo menos não deve se tornar muito severa. É sabido que o paciente sem dor torna-se menos ansioso, participa mais das atividades fisicas e recupera-se melhor.

Com relação a responsabilidade pela avaliação da dor, os enfermeiros colocaram que "a enfermagem é quem avalia se o paciente está com dor porque está mais próxima dele". Pereira ${ }^{(8)}$ menciona que dentre os profissionais que integram a equipe de saúde, o enfermeiro é o que detém o papel fundamental na avaliação da dor dos pacientes. A vantagem de estar em contato diário com os mesmos os coloca em posição única para o desenvolvimento de relacionamentos plenos, os quais possibilitam a obtenção de informações sobre o estado físico e psicológico dos pacientes, essenciais à elucidação de um diagnóstico ainda duvidoso, à implementação de diferentes técnicas analgésicas e à avaliação dos resultados obtidos mediante sua utilização.

Os participantes deste estudo referiram que há pouco conhecimento sobre medidas alternativas comprovadas para alívio da dor e, devido a isso, a equipe de saúde enfrenta limitações para determinar medidas alternativas ou complementares a terapêutica medicamentosa para alívio da dor. O controle da dor pós-operatória parece ter evoluído pouco durante os últimos anos, apesar do reconhecimento da inadequação do seu tratamento, da introdução de novos agentes e técnicas para o tratamento da dor aguda (13) A atuação do enfermeiro pode ser ineficaz no tratamento da dor pós operatória devido ao conhecimento inadequado a respeito da farmacologia dos opiáceos e à preocupação excessiva com o risco do paciente desenvolver hábito e dependência, e se cometer falhas na avaliação da dor dos pacientes (13)
Observou-se que, de maneira geral, os enfermeiros demonstraram estar atentos a dor no pósoperatório de pacientes internados no Centro de Terapia Intensiva. Ressaltaram a importância de sua participação no processo de avaliação da dor e na decisão de condutas que promovam alivio e conforto do paciente. Demonstraram, também, ter consciência das limitações ainda encontradas no processo de alívio da dor, no que se refere a utilização adequada da terapia analgésica, bem como, enfatizaram a importância do aprimoramento de medidas específicas de enfermagem frente a dor. Fica evidente com a realização deste estudo, a necessidade de se buscar, cada vez mais, conhecimentos sobre a experiência dolorosa e as medidas para prevenir e aliviar esse desconforto.

\section{CONSIDERAÇÕES FINAIS}

A avaliação da dor e condutas para alivio

da dor foram as categorias que expressaram como os enfermeiros aliviam a dor de pacientes durante o pósoperatório. Observamos que existe uma preocupação por parte dos enfermeiros no aperfeiçoamento de condutas que visem promover o conforto aliviando a dor e o sofrimento provocado por ela. A avaliação da dor na ótica dos participantes é a conduta inicial para estabelecer ações que previnam e minimizem esse desconforto. Os enfermeiros valorizaram a terapêutica medicamentosa e a complementar ou alternativa para o alívio e prevenção da dor. Salientaram a dependência em relação ao médico no que diz respeito à prescrição adequada de analgésicos, e levantaram a problemática da prescrição ineficaz como conseqüência da subestimação da dor por parte da equipe de saúde.

Dados deste estudo retratam as concepções de Pimenta (12) :"o enfermeiro não é o único profissional a implementar tratamentos de dor, mas detém grande responsabilidade na monitorização da resposta do paciente. Talvez, grande parte da inadequação do tratamento da dor resulte do fato de nenhum profissional isoladamente ser o responsável. Cada um espera que o outro identifique o problema e sugira a solução. Assim, o controle da dor se torna um problema a mais para cada um com baixa prioridade para todos". 


\section{REFERÊNCIAS BIBLIOGRÁFICAS}

(1) Bardin L, Análise de conteúdo. São Paulo: Edições 70, 1994.

(2) Murray MJ, Tratamento da dor na UTI. Olín Ter Int 1990; 2: 247-63,

(3) Leisfer D. Monitorização e registro do controle da dor. Clin Ter Int 1990; 2: 297-308.

(4) Pimenta CA, Koizumi MS, Ferreira MTC, Pimentel ILC. Avaliação da experiência dolorosa. Rev Bras Med, 1995: 74(2): 69-75.

(5) Eisenach JC, Incidência da dor pós-operatória e fatores predisponentes. In: Bonnet F. A dor no meio círúrgico, Porto Alegre: Artes médicas, 1993. p.48-56.

(6) Ready LB, Edwards WT. Tratamento da dor aguda. Rio de Janeiro: Revinter; 1995. Dor pós-operatória no adulto; cap.3, p.21-5.

(7) Melzack R, Wall PD. The challenge of pain. 2 ed. England: Penguin Books, 1991.

(8) Pereira LV. Estimação de categorias e descritores de dor pósoperatória: um enfoque experimental. [dissertação] Ribeirão Preto (SP): Escola de Enfermagem de Ribeirão Preto da Universidade de São Paulo, 1996.

(9) Fortuna A, Fortuna AO. Dor pós-operatória. Rev Bras Cir 1987; 77(5): 291-4.

(10) Kubecka KE, Simon JM, Boettcher JH. Pain manegement knowledge of hospital-based nurses in a rural Appalachian area. J Adv Nurs 1996; 23(5): 861-7.

(11) Bernardo CL. A atuação do enfermeiro no controle da dor. Âmbito Hospitalar 1995; 8(77):23-9.

(12) Pimenta CAM, Koizumi MS, Ferreira MTC, Pimentel ILC, Dor: ocorrência e evolução no pós-operatório de cirurgia cardíaca e abdominal. Rev Paul Enferm 1992; 11(1): $3-10$,

(13) Bonnet F. A dor no meio cirúrgico. Porto alegre: Artes Médicas, 1993.

(14) Sofaer B. Dor manual prático. $2^{\mathrm{a}}$ ed. Rio de Janeiro: Revinter, 1994,

(15) Jimenez SLM. Comfort management: a conceptual framework for exploring issues of pain and comfort. $J$ Perinat Educ 1996; 5(4): 67-70.

(16) Sternbach RA. Clinical aspects of pain. In: Sternback RA. The psychology of pain. $2^{\mathrm{a}}$ ed. New York: Raven Press, 1986. p.223-239.

(17) Gozzani JL. Repercussões orgânicas do quadro doloroso. Rev Bras Med 1994; 51: 6-7.

(18) Bond MR. Dor - natureza, análise e tratamento. $2^{4}$ ed. Rio de Janeiro: Colina, 1986.
(19) Meinhart NT, McCaffery M. Pain: a nursing approach to assessment and analysis. Connecticut: Appleton, 1983.

(20) McCaffery M. Understanding your patient's pain. Nurs 1980; 10(9): 26-31.

(21) Harrinson M, Cotanch PH. Pain: advances and issues in critical care. Nurs Clin North Am 1987: 22(3): 691-7.

(22) Donavan M, Dillon P Incidence and characteristics of pain in a sample of medical-surgical inpatients. Pain 1987; 30(1): 69-78.

\section{Artigo recebido em 23/02/00}

Artigo aprovado em 16/07/01 